\title{
EMcounter-charting the epidemiology of medical emergencies in India: a status report
}

\author{
Satchit Balsari
}

Received: 20 January 2008 / Accepted: 7 February 2008/Published online: 18 March 2008

(C) Springer-Verlag London Ltd 2008

\begin{abstract}
Background In the last decade, the specialty of Emergency Medicine has gained tremendous interest in low and middle income countries, with a demand for new training programs, pre-hospital systems, emergency department expansions and policy change. Yet, little is known about the actual distribution of medical emergencies in these settings. Aim Project EMcounter proposes the implementation of this much needed, uniform, multi-center epidemiologic survey of emergencies in India to provide sound scientific data upon which new training programs, infrastructural expansions, and legislative change can be built.

Methodology A standardized, web-based, user-friendly data entry tool, EMcounter, forms the backbone of this project. The tool is currently piloted at a tertiary center in Chennai, India. The project is aimed at capturing the geographic and temporal variations in over 20 participating centers across the private and public sector in rural and urban India. The
\end{abstract}

Pilot Institution: Emergency Department, Sundaram Medical

Foundation (SMF), Chennai

EMcounter Research Team:

Dr. Satchit Balsari (NYP)

Dr. Parivaran Rajavelu (SMF)

Dr. Dave Anthony (NYP)

Dr. Dean Straff (NYP)

Dr. Nagendra Naidu (SMF)

Dr. Arjun Rajagopalan (SMF)

EMcounter Technical Support:

Ritwik Dey and Mihir Shah

Grants: The pilot phase of the project is supported by a seed grant made by the All India Disaster Mitigation Institute, Gujarat, India.

S. Balsari $(\bowtie)$

Division of Emergency Medicine,

New York-Presbyterian Hospital (NYP),

525 East 68th St,

New York, NY 10021, USA

e-mail: satchitbalsari@gmail.com uniform use of the web-based tool ensures standardization in data collection across the centers. Our pilot project logs patient demographics, pre-hospital transportation, chief complaints, vitals, interventions, disposition and diagnoses. The volume of data thus collected is large and is currently saved in a spread sheet format.

Observations The first quarter has already begun to highlight the epidemiologic differences between a local hospital in Chennai and national averages in the US. The pilot phase has been critical in gauging the robustness of the tool before its expansion to multiple centers and has proved to be invaluable in identifying potential flaws.

Conclusion The early pilot phase has demonstrated that combining the multiple parameters available through the EMcounter database will allow the study of demographics and existing practice algorithms. Expansion of the project to multiple centers will shed objective light on the gaps in healthcare provision at various levels and help design triage and transfer guidelines based on these data. This epidemiologic knowledge can potentially have significant influence in shaping the inventories and designs of emergency departments, and identifying staff needs and skill requirements. The project aims to seek and analyze data that will make the development of emergency medicine in India locally relevant.

Keywords EMcounter Epidemiology · Emergency medicine $\cdot$ India

\section{Emergency medicine in India}

In the last decade, the specialty of Emergency Medicine has gained tremendous interest in low and middle income countries, with a demand for new training programs, prehospital systems, emergency department (ED) expansions 
and policy change. Yet, little is known about the actual distribution of medical emergencies in these settings. Project EMcounter proposes to undertake an epidemiologic study of medical emergencies in India.

In India, the majority of health-care facilities across the country staff its casualty wards or receiving rooms with junior physicians, often recent medical school graduates. These casualty wards are equipped with minimal infrastructure, with almost no provision for critical intervention, often serving as mere triage portals to other areas of the hospital. The need to move away from this traditional approach to providing specialized care early at the footsteps of the hospital is felt across India: several institutions have transformed their traditional casualty wards into specialized emergency departments, large metropolises have launched pre-hospital transport systems, and private institutions have begun training paramedics and emergency physicians. The professionals spearheading these efforts realize the importance of collectively identifying and addressing the challenges faced in developing the specialty of Emergency Medicine in India. For many years, the Society for Emergency Medicine in India (SEMI), in collaboration with emergency physicians from the UK and USA, has been convening annually to explore the regional growth of the specialty. There is universal recognition among key players that the approach must be multi-faceted: legislative change, medical curriculum development, specialty recognition and certification, infrastructure expansion, EMS networking, public awareness and state commitment are all necessary. At this vital juncture, the EMcounter project aims to chart the demography of medical emergencies in India, to provide data critical to the development and local relevance of the specialty.

\section{The rationale for EMcounter}

Data collection is a resource-consuming exercise requiring significant contributions of time, finances and skilled personnel. Consequentially, data gathering is closely related to the need for such data. Infectious diseases have traditionally been responsible for a lion's share of morbidity and mortality in India. The need to understand their incidence, distribution and impact has, over a century, resulted in rich epidemiologic data on infectious diseases in India. Such data have been not only of clinical relevance, but also of vital public health importance, allowing the effective planning and implementation of many preventive programs over the years. ${ }^{1}$

\footnotetext{
${ }^{1}$ The widespread awareness and acceptance of the oral rehydrating solution, the (hopefully) imminent eradication of polio and the (failed) malaria eradication program are some of the larger milestones dotting the Indian public health landscape.
}

The development of Emergency Medicine in India will entail similar data collection to better plan for emergencies presenting to the receiving rooms of hospitals and primary health centers across India. Leading texts in Emergency Medicine and successful Emergency Medical Service models are currently based on contemporary practices in high-income countries. The relative frequencies of various medical emergencies in India almost certainly differ from these high-income nations with their unique population demographics and climes. The exact nature of such differences, however, remains undocumented. Quantification of the nature and extent of these differences would help determine the relative importance and applicability in India of various components of contemporary emergency medicine in high-income settings.

It is therefore imperative that the colossal task of documenting the local epidemiology of emergencies now be undertaken to make the development of the specialty locally relevant. Project EMcounter proposes the implementation of this much needed, uniform, multi-center epidemiologic survey of emergencies in India to provide sound scientific data upon which India's emergency medicine training programs, infrastructural expansions and legislative changes can be built.

\section{Methodology}

EMcounter aims to capture the geographic and temporal variations in medical emergencies presenting to over 20 participating centers across the public and private sector in rural and urban India. At each center, epidemiologic data will be collected for a period of 365 days. To ensure uniformity in data collection across the participating centers, we have designed a standardized, user-friendly data entry tool, EMcounter, which forms the backbone of this project. In the following sections, we share our experiences with piloting EMcounter, the lessons learned and the steps ahead.

\section{EMcounter: The web-based tool}

The EMcounter tool is hosted at our homepage www. EMcounter.com The tool is password-protected and generates a unique case number for each patient. Only the local institution can trace this number to the patient's hospital registration number. No patient identifiers are logged into the EMcounter tool.

Most hospitals that we aim to cover do not have electronic medical record systems. This makes data collection a laborious process and the need for meticulousness imperative. In order to limit the number of entries per patient encounter, we have limited the recordable data points to only those that have evident relevance to the 
project goal of charting the epidemiology of medical emergencies. The tool contains easy, searchable, drop-down menus, containing exhaustive lists of drug inventories and diagnostic tests adapted to local nomenclature. The backend database retains generic terms to allow for standardization across the centers during analysis.

Our pilot project logged patient demographics, prehospital transportation, chief-complaints, vitals, interventions, disposition and diagnosis. If the patients were not discharged home from the ED, final diagnosis at discharge from the hospital was recorded. The large number of data points thus collected are currently saved in a spread-sheet format.

\section{The pilot}

The project is currently piloted at the Sundaram Medical Foundation (SMF), Chennai, a leading, 160-bed, not-forprofit, postgraduate teaching hospital. The ambulatory services at the hospital see about 180,000 patient visits. The hospital admits 11,000 patients annually.

The Emergency Department at SMF is open 24-h a day, every day of the year, and recruits approximately $30 \%$ of the hospital's annual admissions. It has seven "regular" beds, equipped with vital signs monitoring capabilities, and one resuscitation room (with two "CPR beds"), fully equipped with monitors, a crash cart, suture and IV carts and a code blue system to activate the "crash team." The ED provides 24-h imaging facilities, including computerized tomography. The ED has easy access to three operation rooms; the hospital's ICU has eight beds. The ED is always staffed by doctors, nurses and technicians. The physicians in the ED are usually recent medical graduates on a 3-6month rotation, and are supervised by consultants and postgraduates from other departments, especially the ICU. All tests and treatments need supervisory clearance.

A prospective, observational test run of the EMcounter tool was completed in January 2007, and appropriate adjustments were made to the tool and to the paper records in the ED. The tool's 365-day run was launched in June 2007. Data are being culled daily from the paper records of all patients presenting to the ED and uploaded online to the EMcounter tool. Records of admitted patients are electronically flagged and followed to discharge. Findings from the test run were presented at the ACEP 2007 Scientific Assembly in Seattle, WA, and findings from the first quarter of the pilot run at the EMCON 2007 conference in Chennai, India.

\section{Observations}

The first quarter has already begun to highlight the epidemiologic differences between a local hospital in Chennai and national averages in the US (from where multiple institutions, including ours, are nurturing educational and training collaborations with hospitals in India). Early analysis revealed predictable, but hitherto un-quantified trends: fever and gastroenteritis constituted over a quarter of the presentations to the Chennai ED; lacerations, upper respiratory infections, vertigo, urinary tract infections and asthma constituted the other leading chief complaints. Chest pain did not make it to the top ten in the first quarter. In fact, of all the patients that presented to the ED, only $5.7 \%$ reported chest pain: $82.5 \%$ of these patients with chest pain were discharged; of the 29 admitted, 14 patients went to the ICU, 12 of whom were diagnosed with acute coronary syndrome.

\section{Limitations}

The pilot phase has been critical in gauging the robustness of the tool before its expansion to multiple centers, allowing us to address the limitations unearthed by the initial run. For example, the tool logged all prescribed interventions, irrespective of implementation. All imaging studies ordered were recorded. But whether the patient could in fact afford the study (a question wholly relevant in the absence of EMTALA-like legislation) was not recorded. If the elderly patient who had blunt head injury from a fall went home from the ED, we could not tell if the discharge was against medical advice, or subsequent to a negative CT and observation, or after a declined (unaffordable) CT request. Our "diagnosis" field failed to differentiate new diagnoses from existing co-morbidities: were all the patients admitted to the ICU with diabetes truly admitted for diabetic ketoacidosis, or was diabetes merely a confounding co-morbidity? We did not know. The pilot run has indeed proved invaluable in identifying our Achille's heel(s).

\section{Future potential}

On the upside, the daily data entry during the pilot phase has demonstrated the potential of EMcounter as a dynamic surveillance tool in the ED-a prospect that has peaked the interest of local state officials. The computing and information technology at our disposal in 2008 make the collection and interpretation of the large data sets that EMcounter aims to capture plausible. The first quarter has demonstrated that combining the multiple parameters available through EMcounter will allow the study of multiple practice algorithms: Does every smoker with a cough and fever get a chest X-ray? Were all tender anatomical snuff-boxes with "negative X-rays" splinted? Are the existing triage systems validated by final diagno- 
ses? Asking and answering such questions will help the specialty of emergency medicine in India meet local needs, develop locally relevant curricula, hone locally needed skill sets and address local resource constraints before embracing foreign decision-making algorithms.

\section{Next steps}

The EMcounter project is now in the process of recruiting partners elsewhere in the country. The project requires dedicated personnel for data entry and part-time researchers at each local institution. Each participating institution will be required to acquire approval or exemption from its Institutional Review Board (IRB). Institutions that do not have access to an IRB will be expected to adhere to IRB guidelines formulated by collaborating institutions. Data entry precision will be monitored through ad hoc checks conducted by independent reviewers that will be allowed access to the original paper records for comparison with EMcounter data-entries. The final EMcoutner database is accessible to all participating institutions, with institutional identifiers blinded on request.

The EMcounter technical team will add an Analysis Component to the tool in mid 2008 to help query the database in a user-friendly interface and present data in a dynamic, multi-media format for interpretive ease. The tool is now ready for expansion to multiple locations.

\section{Outcomes}

This project recognizes the work of the many individuals and agencies involved in promoting the science of emergency medicine in India and seeks to contribute to this local effort by providing critically needed data that will help shape the curriculum of emergency medicine training. The epidemiologic knowledge will also better shape the inventories and designs of emergency departments and identify staff needs and skill requirements.

The project will shed objective light on the gaps in health-care provision at various levels and help design triage and transfer guidelines based on these data. The data will identify the demographic patterns of diseases prevalent in the participating regions and their annual cyclical trend.

The creation of successful people-centric emergency medicine systems will require the consecrated efforts of many agencies in India over a sustained period of time. This project is aimed at making a meaningful contribution to that end. 